\title{
Tratamento Farmacológico e Não Farmacológico no Manejo da Dor de Pacientes em Pós-Operatório Imediato (POI)
}

\author{
Sabrina da Silva Nascimento, ${ }^{1}$ Gabriela Elisa Hirsh, ${ }^{2}$ Carolina Renz Pretto, ${ }^{2}$ \\ Christiane de Fátima Colet, ${ }^{2}$ Eniva Miladi Fernandes Stumm²
}

\begin{abstract}
RESUMO
O objetivo deste estudo foi analisar evidências científicas referentes às modalidades farmacológicas e não farmacológicas utilizadas no manejo da dor de pacientes em Pós-Operatório Imediato (POI) de cirurgias em geral. Este trabalho é uma revisão integrativa da literatura realizada nas bases Lilacs, Medline/Pubmed, Scopus e Web of Science, entre 2002 e 2017, que resultou na análise de 38 artigos científicos. Dos artigos analisados, $71 \%$ abordaram métodos farmacológicos e $28 \%$ medidas não farmacológicas para o tratamento da dor no POI. O manejo adequado da dor é de responsabilidade dos profissionais envolvidos no cuidado e os hospitais devem dispor de equipes e procedimentos para alívio da dor visando a diminuir efeitos colaterais, tempo de hospitalização e acelerar a recuperação do paciente. Os métodos não farmacológicos podem ser uma modalidade complementar promissora para melhorar a dor no pós-operatório, pois são técnicas acessíveis e fáceis de serem executadas e quando praticada por profissionais capacitados, os eventos adversos ocorrem raramente.
\end{abstract}

Palavras-chave: Manejo da dor. Cuidados pós-operatórios. Tratamento farmacológico. Terapias complementares. Eventos adversos.

PHARMACOLOGICAL AND NON-PHARMACOLOGICAL TREATMENT IN PAIN MANAGEMENT

OF PATIENTS IN IMMEDIATE POSTOPERATIVE PERIOD (IPP)

\section{ABTRACT}

The objective of this study was to analyze scientific evidences referring to the pharmacological and non-pharmacological modalities used in the management of the pain of patients in the immediate postoperative period (IPP) of surgeries in general. This is an integrative review of the literature in which a search of articles in the Lilacs, Medline/Pubmed, Scopus and Web of Science databases was conducted between 2002 and 2017, which resulted in the analysis of 38 scientific papers. Among analyzed articles, $71 \%$ approached pharmacological methods and $28 \%$ non-pharmacological measures for the treatment of pain in the IPP. Proper pain management is the responsibility of the professionals involved in care and hospitals should have teams and procedures for pain relief to reduce side effects, hospitalization time and accelerate patient recovery. Non-pharmacological methods may be a promising complementary modality to improve post-operative pain, as these techniques are accessible and easy to perform and when practiced by trained professionals, adverse events rarely occur.

Keywords: Pain management. Postoperative care. Pharmacological treatment. Complementary therapies. Adverse events.

ACEITO EM: 10/10/2019

MODIFICAÇÕES SOLICITADAS EM: 23/11/2019

RECEBIDO EM: 6/7/2020

\footnotetext{
Autora correspondente. Universidade Regional do Noroeste do Estado do Rio Grande do Sul (Unijuí) - Campus ljuí. Rua do Comércio, 3000, bairro Universitário. 98700-000, ljuí/RS, Brasil. http://lattes.cnpq.br/7543008599417369. sasanascimento8@hotmail.com
}

2 Universidade Regional do Noroeste do Estado do Rio Grande do Sul (Unijuí) - Campus ljuí. ljuí/RS, Brasil. 


\section{INTRODUÇÃO}

A dor é um evento comum, expressa de formas diferentes e sentida por todos os seres humanos, portanto sua avaliação, mensuração e controle são importantes para o cuidado com o paciente (MORO; BRILHA; MARIANO, 2012). Sua ocorrência é frequente em pacientes submetidos a procedimentos cirúrgicos e no Pós-Operatório Imediato (POI), período posterior ao procedimento anestésico/cirúrgico, o qual se inicia na sala cirúrgica, quando ocorre reversão da anestesia, estendendo-se até 2 a 3 horas e pode durar até 24 horas após o término do procedimento (ROCHA; MORAES, 2010; MOREIRA et al., 2013). A Dor Pós-Operatória (DPO) é subjetiva, pessoal e compreendida como um evento natural de proteção do corpo, o qual ocorre sempre que algum tecido é lesionado e desencadeia estímulo doloroso (SMELTZER et al., 2009; IUPPEN; SAMPAIO; STADÑIK, 2011).

O controle inadequado da dor em pacientes no POI é responsável por inúmeras repercussões e aumenta o tempo de internação e custos hospitalares (MORO; BRILHA; MARIANO, 2012; MEISSNER et al., 2015). Para esse controle ser realizado, medidas farmacológicas e não farmacológicas podem ser utilizadas. Entre as medidas farmacológicas destaca-se uso de anti-inflamatórios e analgésicos, principalmente da classe dos opioides, mas seu uso inadequado está associado à dependência e até mortes por overdose, tornando este um problema de saúde (TAN; LAW; GAN, 2015; TICK et al., 2018). Como uma alternativa para este tipo de tratamento, medidas não farmacológicas têm sido estudadas. Elas envolvem terapias físicas que ativam o sistema sensitivo-discriminativo e estimulam o sistema supressor da dor, como aplicação de calor/frio, massagem, acupuntura, entre outras; e técnicas cognitivo-comportamentais que atuam interferindo no estímulo doloroso por meio do relaxamento muscular, distração da atenção e sugestão, e envolve, entre outras, imaginação dirigida e hipnose (PIMENTA, 1990; TAN; LAW; GAN, 2015).

Evidências apoiam o uso de técnicas farmacológicas como parte da analgesia multimodal, mas existem posicionamentos contraditórios referentes à eficácia das técnicas não farmacológicas (KOMANN et al., 2019). Diante do exposto, este estudo tem como objetivo analisar evidências científicas referentes às modalidades farmacológicas e não farmacológicas utilizadas no manejo da dor de pacientes em POI de cirurgias em geral.

\section{METODOLOGIA}

Trata-se de uma revisão integrativa da literatura, estruturada em seis etapas: formulação da pergunta e objetivo, amostragem, extração de dados dos estudos primários, avaliação crítica, análise e síntese dos resultados e apresentação da revisão integrativa. Definiu-se a seguinte questão norteadora: 0 que tem sido evidenciado na literatura científica referente às modalidades farmacológicas e não farmacológicas utilizadas no manejo da dor de pacientes em POI de cirurgias em geral?

A busca de artigos ocorreu nas bases de dados eletrônicas: Literatura Científica da América Latina e do Caribe em Ciências da Saúde (Lilacs), Medical Literature Analysis and retrieval System Online via US National Library of Medicine - National Institutes of Health (Medline/Pubmed), Web of Science (coleção principal) e Sci Verse Scopus (Scopus). O período de coleta dos artigos compreendeu os meses de novembro a dezembro de 2017, com as seguintes combinações de descritores e sinônimos: ((("Pain,postoperative" OR "postoperative pain") AND ("pain management" $O R$ "pain management")) AND ("drug therapy" OR "therapy,drug" OR pharmacotherapy)) e ((("Pain,postoperative" OR "postoperative pain") AND ("pain management" $O R$ "pain management")) $A N D$ ("complementary therapies" $O R$ "therapy, complementary" OR "therapies, complementary" OR "alternative medicine" $O R$ "alternative therapies" OR phytotherapy $O R$ reflexotherapy $O R$ "acupuncture therapy" $O R$ "flower essences" OR "relaxation therapy")). Os termos nortearam-se nos Descritores em Ciências da Saúde (DeCS) e Medical Subject Headings (MeSH). Os dados do cruzamento dos DeCS e MeSH resultaram em 911 artigos, nos quais foram aplicados os critérios de seleção elencados para esta avaliação.

Os critérios de inclusão elencados foram: artigos originais disponibilizados na íntegra on-line, em Português, Espanhol e Inglês, publicados a partir de 2002, ano em que o Ministério da Saúde (MS) instituiu o Programa Nacional de Assistência à Dor e Cuidados Paliativos, até dezembro de 2017; informações no título ou resumo que indicassem ter como participantes da pesquisa pacientes submetidos à cirurgia e em POI (até 24 horas após cirurgia). Os critérios de exclusão foram: pesquisas com crianças e animais, estudo de caso, cirurgias ambulatoriais, estudos com nível de evidência 5, 6 ou 7 de acordo com Melnyk e Fineout-Overholt (2011) e artigos não completos na base de dados, dados resumidamente apresentados na Figura 1. 
Figura 1 - Diagrama da seleção de artigos para revisão integrativa

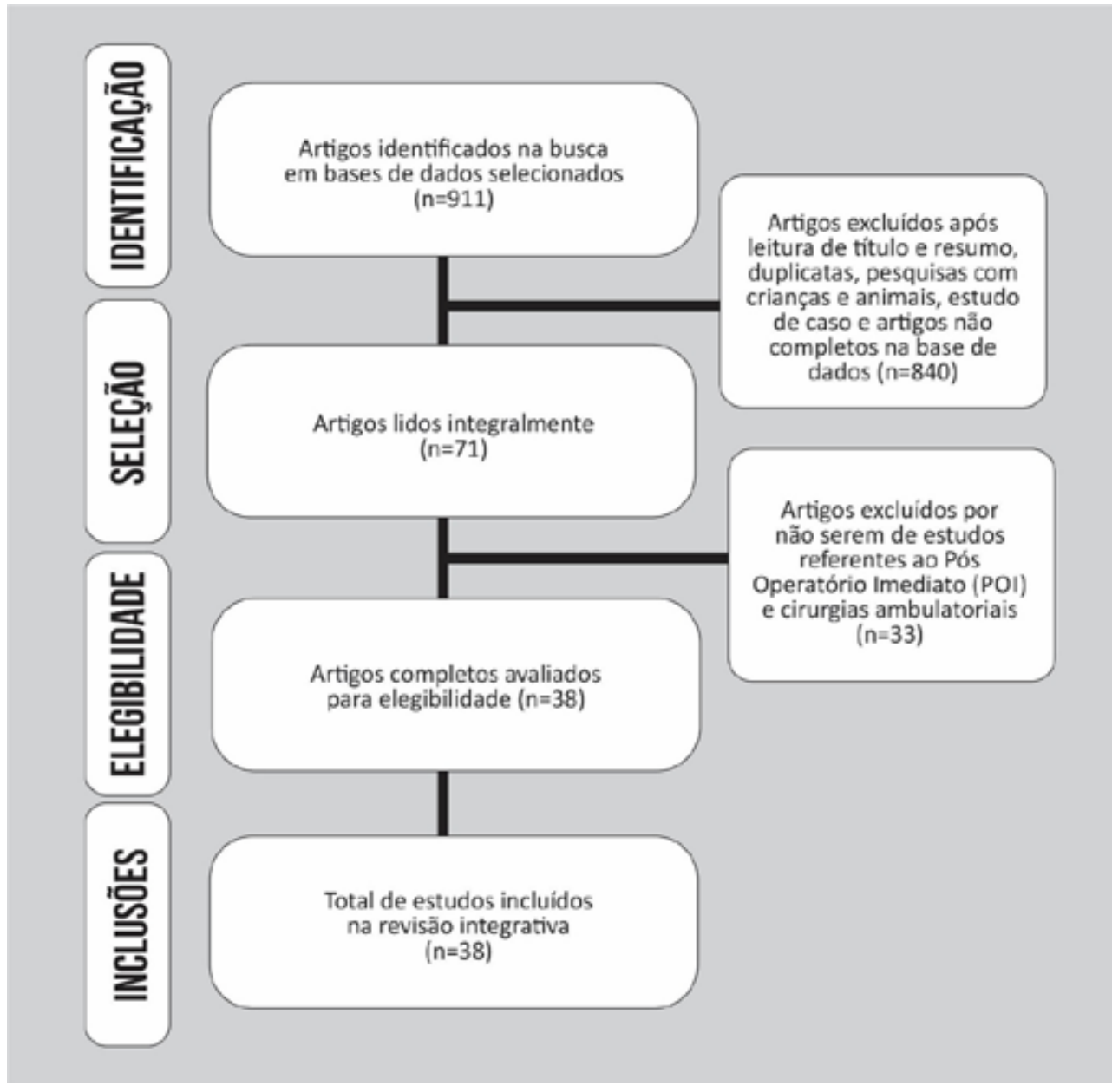

Fonte: Autoras da pesquisa, 2018.

Tabela 1 - Quantitativo (n) dos artigos encontrados (E) e selecionados (S) após revisão integrativa por base de dados

\begin{tabular}{|c|c|c|c|c|}
\hline \multirow[t]{2}{*}{ BASE DE DADOS } & \multicolumn{2}{|c|}{$\begin{array}{c}\text { DeCS/MeSH } \\
\text { "Pain,postoperative" OR "postoperative } \\
\text { pain AND "pain management" OR } \\
\text { "pain management) AND "drug } \\
\text { therapy" OR "therapy,drug" OR } \\
\text { pharmacotherapy }\end{array}$} & \multicolumn{2}{|c|}{$\begin{array}{c}\text { DeCS/MeSH } \\
\text { "Pain,postoperative" OR "postoperative pain" } \\
\text { AND "pain management" OR "pain management" } \\
\text { AND "complementary therapies" OR "therapy, } \\
\text { complementary" OR "therapies, complementary" OR } \\
\text { "alternative medicine" OR "alternative therapies" OR } \\
\text { phytotherapy OR reflexotherapy OR "acupuncture } \\
\text { therapy" OR "flower essences" OR "relaxation therapy" }\end{array}$} \\
\hline & $\mathrm{E}$ & $S$ & $\mathrm{E}$ & $S$ \\
\hline Lilacs & 03 & 01 & 0 & 0 \\
\hline Medline/Pubmed & 346 & 13 & 119 & 02 \\
\hline Scopus & 308 & 13 & 97 & 07 \\
\hline \multirow[t]{3}{*}{ Web of Science } & 24 & 0 & 14 & 02 \\
\hline & Total: 681 & Total: 27 & Total: 230 & Total: 11 \\
\hline & \multicolumn{4}{|c|}{$\begin{array}{l}\text { Total encontrado: } 911 \\
\text { Total selecionado: } 38\end{array}$} \\
\hline
\end{tabular}

Fonte: Autoras da pesquisa, 2018.

A seleção preliminar foi realizada pelo pesquisador principal a partir da leitura do título e dos resumos dos artigos, com a aplicação dos critérios de seleção. Foram selecionados para leitura integral 71 artigos e destes, 33 foram excluídos por não abordarem a avaliação da dor no POI até 24 horas após cirurgia ou pelo fato de os pacientes terem sido submetidos a procedimentos ambulatoriais, conforme descrito nos critérios 
de seleção. Ao final da seleção, 38 artigos integraram a análise, cuja avaliação crítica e categorização de evidências ocorreram em conjunto com outro revisor, conforme dados descritos na Tabela 1.

Para categorização das evidências, foram considerados quatro dos sete níveis de evidência preconizados por Melnyk e Fineout-Overholt (2011) para intervenções na área da saúde: nível 1, as evidências são provenientes de revisão sistemática ou meta-análise de ensaios clínicos randomizados controlados ou oriundas de diretrizes clínicas baseadas em revisões sistemáticas de ensaios clínicos randomizados controlados; nível 2, evidências derivadas de pelo menos um ensaio clínico randomizado controlado; nível 3, evidências obtidas de ensaios clínicos sem randomização; nível 4, evidências provenientes de estudos de coorte ou de caso-controle bem delineados.

A análise dos resultados foi realizada em torno de três polos cronológicos: pré-análise, exploração do material, tratamento e interpretação dos resultados (BARDIN, 2011). Após leitura dos artigos selecionados na íntegra organizou-se a análise das temáticas, para melhor descrever e classificar os resultados, com base nas evidências científicas sobre o tema estudado.

\section{RESULTADOS}

Dos 38 artigos que integraram a amostra, 8 (21\%) eram nível de evidência 1, uma revisão sistemática ou meta-análise de ensaios clínicos randomizados controlados; 25 (65,8\%) nível 2, derivados de ensaio clínico randomizado controlado; 2 (5,2\%) nível 3 obtidas de ensaios clínicos sem randomização e $3(8 \%)$ nível 4 provenientes de estudos de coorte ou de caso-controle delineados. Destes, 37 (97\%) estavam no idioma Inglês e $1(3 \%)$ em Português. Os periódicos com maior número de publicações foram Pain Medicine e Plos One, ambos com $3(15,7 \%)$ artigos cada.

Quanto ao local das pesquisas, 6 (32\%) foram realizadas nos Estados Unidos e na China, 4 (21\%) no Irã e na Coreia, $3(8 \%)$ na Turquia, $2(10 \%)$ na Grécia e Taiwan e as demais na França, Egito, Finlândia, Espanha, Inglaterra, Itália, Israel, Noruega, Alemanha, Suécia e Tailândia (29\%). Com relação ao ano de publicação, em 2015 foram publicados 11 (28\%) artigos, constituindo este o com maior número de publicações.

Entre os estudos, 27 (71\%) abordaram métodos farmacológicos para o tratamento da dor no POI. Os medicamentos avaliados foram paracetamol e cetamina em 4 (15\%) estudos cada, dexametasona, bupivacaína, lonorxicam e morfina em 3 (11\%), fentanil, pregabalina, dexmedetomidina, levobupivacaína e ropivacaína em $2(7 \%)$ e cetorolaco, diclofenaco, flurbiprofeno, gabapentina, ibuprofeno, meperidina, parecoxibe, piroxicam, remifentanil, oxicodona e tramadol em 1 (3\%). A analgesia multimodal foi citada em 11 $(23,6 \%)$ pesquisas e mostrou-se eficaz na diminuição do uso de opioides, na avaliação da escala de dor visual e diminuiu o tempo de permanência dos pacientes no hospital.

Por outro lado, o uso de medidas não farmacológicas para o tratamento da dor no POI foi analisado em $11(29 \%)$ investigações. A acupuntura foi utilizada por $4(36 \%)$ delas, seguida de aromaterapia, massagem reflexológica, estimulação nervosa elétrica transcutânea de alta frequência, relaxamento, massagem de pés e mãos, e terapia da ansiedade, a acupressão auricular, em 1 manuscrito (9\%) cada.

Alguns estudos (20\%) avaliaram diversas cirurgias, concomitantemente. Os procedimentos cirúrgicos abordados foram artroplastia de joelho em 4 (10\%) estudos, cesariana em $3(7 \%)$, histerectomia, amigdalectomia em 2 (5\%) estudos cada, tireoidectomia em 2 (5\%) e artroplastia de ombro, craniotomia, artroscopia do manguito rotador, colecistectomia, laparoscopia de câncer colorretal, toracotomia, nefrolitotomia percutânea, reposição unilateral total do quadril, nefrectomia, abdômem, dissecção endoscópica de neoplasia gástrica, laparoscopia renal e adrenal, apendicectomia, pancreática, coluna, costas, urogenital e cirurgia ginecológica em 1 (3\%) estudo cada. As amostras elencadas dos estudos variaram de 40 a 865 participantes.

\section{DISCUSSÃO}

A partir da leitura e análise dos artigos selecionados, emergiram duas categorias analíticas, descritas e analisadas a seguir:

\section{Categoria 1 - Modalidades farmacológicas para manejo da dor em pacientes no POI}

A terapia para o tratamento da dor é ampla e a escolha adequada do fármaco é fundamental para tratar a DPO de forma efetiva. O uso de opioides como analgésicos tem vários efeitos que podem prolongar a internação. Assim, a analgesia multimodal tornou-se fundamental para recuperação do paciente no $\mathrm{PO}$, alternativa promissora, que pode reduzir dependência e necessidades de altas doses de medicamentos analgésicos, reduzindo assim os riscos de efeitos colaterais associados a tal procedimento (FLETCHER; SPERA, 2012; TAN; LAW; GAN, 2015). 
Embora a analgesia multimodal tenha sido recomendada como um tratamento ideal para DPO, apenas alguns ensaios clínicos testaram a hipótese de que a combinação de diferentes analgésicos não opioides exerce melhores efeitos analgésicos do que os medicamentos isolados. Diante disso, um estudo com 148 pacientes testou a combinação de gabapentina, cetamina, lornoxicam e ropivacaína local e mostrou que a referida técnica não proporcionou analgesia superior à da gabapentina ou ao lornoxicam isolados, nas primeiras 24 horas após colecistectomia laparoscópica, apenas a frequência de náuseas pós-operatórias (KOTSOVOLIS et al., 2015).

A analgesia preventiva é uma forma de intervenção da dor antes da estimulação nociva e tem sido indicada com o objetivo de aliviar a DPO, podendo ser associada à terapia multimodal (KHALILI; JANGHORBANI; SARYAZDI, 2013). Nesse sentido, um estudo verificou se a combinação de analgesia multimodal e analgesia preventiva (analgesia preventiva multimodal) poderiam ter melhor efeito analgésico no manejo da dor e redução de efeitos colaterais (CHEN, et al., 2015). Tal estudo avaliou a analgesia preventiva multimodal com flurbiprofeno, fentanil e cetamina em cem pacientes submetidos à histerectomia transabdominal e evidenciou que a analgesia preventiva multimodal pode reduzir significativamente a escala analógica visual (EVA), inibir a resposta ao estresse e reduzir a resposta inflamatória nesses pacientes, o que pode ser uma estratégia racional para o controle da dor. Além disso, Santoso et al. (2014) mostraram que o controle da dor multimodal esteve associado à hospitalização significativamente reduzida após histerectomia abdominal aberta, revelando mais um benefício dessa terapia.

O gerenciamento de dor multimodal também mostrou ser benéfico quando aplicado à cirurgia ortopédica. A aplicação de bupivacaína lipossomal, solução salina e marcaína na ferida operatória, e dexametasona intravenosa durante a artroplastia do ombro, em 55 pacientes, reduziu o tempo de permanência hospitalar, uso de opioides e EVA (ROUTMAN et al., 2017). Por outro lado, estudo retrospectivo não demonstrou benefício da bupivacaína lipossomal para DPO em 120 pacientes submetidos à artroplastia total de joelho (WHITE et al., 2015). Os autores explicam que esses resultados podem ter sido obscurecidos pelo aumento do uso de analgésicos adjuntos entre os pacientes do grupo controle, pois a bupivacaína lipossomal pode oferecer várias vantagens sobre a terapia regional, que incluem facilidade de administração, redução do tempo de enfermagem referente ao monitoramento e melhor satisfação do paciente.

Estudo prospectivo com 50 pacientes submetidos à cirurgia artroscópica de manguito rotador avaliou eficácia e segurança a curto prazo da administração de analgésicos multimodais, associada a corticosteroides, ao final da cirurgia (PERDREAU; JOUDET, 2015) e demonstrou que esta associação reduziu a DPO imediata, uso de morfina e tempo de recuperação, sem risco aparente de infecção. Os autores pontuam que resultados desta prática requerem avaliação em longo prazo, especialmente no que respeita à reabilitação funcional e à recuperação do tendão.

Em outro estudo com 66 pacientes submetidos à cirurgia laparoscópica renal e adrenal transperitoneal, a administração intravenosa de paracetamol e lornoxicam foi mais eficaz do que a infiltração de levobupivacaína. Além disso, os pacientes tratados com este medicamento precisaram de doses superiores de tramadol intravenoso, com o uso da Analgesia Controlada pelo Paciente (PCA) e administração adicional de petidina, quando comparados com grupos nos quais foram administrados paracetamol e lornoxicam (TUNCEL et al., 2015), corroborando os benefícios da terapia multimodal.

Estudo com 66 pacientes avaliou a eficácia do uso associado de levobupivacaína, paracetamol e meperidina no tratamento da DPO e mostrou que a quantidade e a frequência de opioides usados, os efeitos colaterais e o escore EVA eram menores com o uso desta associação quando comparados ao uso de fármacos isolados, mostrando que a anestesia multimodal é segura e eficaz como método de analgesia em pacientes submetidos à nefrolitotomia percutânea (GOKTEN et al., 2011). Em dois estudos com 90 e 865 pacientes, respectivamente, o paracetamol intravenoso como complemento à analgesia multimodal e o parecoxibe intravenoso combinado com bloqueio femoral mostraram-se igualmente eficazes na redução da DPO e do consumo de opioides após artroplastia total de joelho (SARRIDOU et al., 2015; LIANG et al., 2017).

Outro aspecto importante da anestesia multimodal é o uso de adjuvantes com o objetivo de prolongar a anestesia espinhal e retardar o aparecimento da DPO, além de reduzir as doses e frequência de analgésicos (PARK; JEON, 2016). A referida pesquisa foi desenvolvida com 44 pessoas e mostrou que 150 mg de pregabalina oral, administrada 2 horas antes da anestesia espinhal, prolongou ambos os bloqueios sensoriais e motores induzidos pela anestesia da bupivacaína. Quanto ao tempo para o primeiro pedi- 
do de analgésicos $\mathrm{PO}$, este, conforme os autores, foi prolongado e aliado a menores doses de analgésicos opioides nas primeiras 24 horas subsequentes à cirurgia urogenital. Uma meta-análise de 6 ensaios clínicos com 452 paciente avaliou a pregabalina no controle da DPO aguda após cirurgia ginecológica e mostrou efeito analgésico e poupador de opioides e diminuiu a frequência de efeitos adversos durante as primeiras 24 horas após cirurgia ginecológica (YAO; SHEN, ZHONG, 2015).

Arslan et al. (2011) avaliaram a eficácia do paracetamol e lornoxicam intravenoso na analgesia pós tireoidectomia e redução do consumo de tramadol em 60 pacientes. 0 estudo mostrou que a administração intravenosa de lornoxicam e paracetamol diminuiu o escore de DPO, necessidade de opioides e incidência de náuseas, vômitos e prolongamento do tempo para primeira readministração analgésica. Outro estudo com o mesmo número de participantes submetidos à tireoidectomia avaliou se a infusão intraoperatória de alta dose de remifentanil poderia atenuar a DPO e encontrou que altas doses de remifentanil diminuíram os escores EVA e reduziram o consumo de morfina no PO (ZHANG et al., 2014).

A dor pós-toracotomia é considerada uma das mais intensas e vários métodos e novos estudos estão em desenvolvimento para amenizá-la (MERCANOĜLU et al., 2013). Um estudo comparou a eficácia e efeitos colaterais de morfina intravenosa (MIV), morfina epidural (ME), morfina epidural associada à bupivacaína (MEB) e morfina epidural associada à ropivacaína (MER) para tratamento da dor em 60 pacientes. Concluiu-se que a ME em infusão, com a técnica de PCA foi mais eficaz e preferida pelos participantes da pesquisa. Kim et al. (2016) avaliaram a eficácia da morfina intratecal para controle de DPO em 45 pacientes com carcinoma de células renais submetidos à nefrectomia aberta e constataram que uma única injeção intratecal de morfina combinada com PCA proporcionou analgesia PO mais eficaz do que PCA isolada, além de reduzir os requisitos de opioides por até 24 horas.

Pesquisa comparou os efeitos analgésicos da administração de tramadol e piroxicam na DPO após parto cesário em 160 pacientes (FARSHCHI; GHIASI, 2010). Os autores avaliaram grau de dor, náuseas e vômitos e constataram que a intensidade da dor diminuiu acentuadamente ao longo do tempo em ambos os grupos e os efeitos colaterais foram mínimos com todos os tratamentos. Eles concluíram que a administração aliviou a DPO, reduziu a necessidade do uso de analgésicos opioides com menos efeitos colaterais nas primeiras 24 horas do PO. Outro estudo com cem pa- cientes após cesariana investigou a eficácia e os efeitos colaterais do supositório de morfina em comparação com o supositório de diclofenaco no tratamento da DPO e mostrou que o supositório de diclofenaco retardou o uso de petidina em $5,24 \pm 4,07 \mathrm{~h}$. Já o supositório de morfina postergou em $3,28 \pm 2,16 \mathrm{~h}$ o uso de petidina, com diferença significativa entre os grupos (MAHDAVI et al., 2016).

$\mathrm{O}$ sistema transdérmico iontoforético de fentanil (Fentanil ITS) mostrou ser seguro e eficaz no tratamento da dor aguda de vários tipos de cirurgia maior (HARTRICK et al., 2006). Eles compararam a eficácia, segurança e conveniência do fentanil ITS associado com analgesia controlada pelo paciente e morfina intravenosa para o tratamento da dor aguda, em 799 pacientes, após a reposição unilateral total do quadril e concluíram que ambas as técnicas apresentaram resultados de analgesia semelhantes nas primeiras 24 horas de tratamento.

Uma vez que o tratamento da dor com um fármaco poupador de opiáceos, como a cetamina, pode melhorar a analgesia e diminuir os efeitos adversos, uma revisão sistemática com 37 ensaios para avaliar a eficácia e tolerabilidade da cetamina administrada perioperatoriamente no tratamento de DPO aguda em adultos foi realizada (BELL et al., 2006) e os resultados mostraram que os efeitos adversos associados a esse medicamento foram leves ou ausentes e que ele é eficaz na redução da náusea, vômito e dos requisitos de morfina nas primeiras 24 horas após a cirurgia, quando comparados com placebo ou morfina. Outra investigação também mostrou que doses subanestésicas de cetamina reduziram requisitos de opioides, mas a dosagem ideal não foi definida (FREDHEIM; BORCHGREVINK; KVARSTEIN, 2011). Os autores dessa investigação afirmaram que o efeito de um analgésico depende de quais fármacos estão incluídos no tratamento da dor e que o uso de múltiplos medicamentos e técnicas são necessários para o equilíbrio entre o alívio da dor, os efeitos colaterais e o risco.

A dexmedetomidina foi citada em dois estudos e relacionada com procedimentos cirúrgicos distintos, com vistas a fornecer analgesia efetiva para reduzir as doses de morfina no PO sem aumentar a incidência de efeitos colaterais (ARAIN et al., 2004; OHTANI, et al., 2011). Em 76 pacientes submetidos à craniotomia, a infusão de dexmedetomidina reduziu a DPO e o consumo de analgésico, além de proporcionar tempo mais curto para a extubação e reduzir náusea e vômitos nas primeiras 24 horas após cirurgia, sem aumentar riscos de bradicardia ou sedação excessiva (PENG et al., 2015). Estudo com 45 pacientes em PO de cirur- 
gia laparoscópica de câncer colorretal mostrou que a administração intraperitoneal de dexmedetomidina 1 $\mu \mathrm{g} / \mathrm{kg}$ combinada com bupivacaína melhorou a qualidade, duração da analgesia PO e efeito poupador de analgésico quando comparado com bupivacaína isolada (FARES, et al., 2015).

Os efeitos clínicos da dexametasona pré e pós-operatória foram avaliados em 40 pacientes com neoplasia gástrica após a dissecção de submucosa endoscópica (PYO et al., 2016). Os resultados mostraram que a administração pré-operatória pode produzir efeito analgésico superior quando comparada com a administração pós-operatória deste medicamento. No tratamento da dor de 90 pacientes após amigdalectomia, a dexametasona reduziu o edema e DPO secundária, já a aplicação de oxicodona reduziu significativamente a DPO (VAIMAN; KRAKOVSKI; HAITOV, 2011).

Outra opção para analgesia no POI são os Anti-Inflamatórios Não Esteroides (Aines) frequentemente utilizados como componentes da terapia multimodal para o tratamento da DPO, pois diminuem o consumo e efeitos colaterais relacionados aos opioides, mas seu uso está limitado devido a seus eventos adversos, como sangramento cirúrgico e úlcera (DIRKMANN et al., 2015). Assim, um estudo com 206 pacientes avaliou a eficácia e segurança de uma nova formulação de ibuprofeno intravenoso para o tratamento da DPO em uma população europeia. A administração perioperatória de $800 \mathrm{mg}$ de ibuprofeno a cada 6 horas em cirurgia abdominal diminuiu o uso de morfina e o escore de dor, e mostrou-se seguro e tolerado pelos pacientes, com benefício analgésico e redução de riscos associados ao consumo de opioides (MARTíNEZ et al., 2016).

O cetorolaco é um Aine de início rápido e pode ser usado tanto para administração intraoperatória quanto para tratamento da DPO e sua eficácia no controle da dor aguda após a cirurgia abdominal foi bem documentada. Assim, um estudo com 80 pacientes avaliou se havia diferenças no alívio da dor com o uso de cetorolaco via endovenosa comparado à infusão contínua e em horários preestabelecidos (RUSSO et al., 2012) e mostrou que a administração a cada 8 horas ofereceu maiores benefícios em relação à infusão contínua do medicamento.

Com base nos artigos analisados, pensa-se que o manejo adequado da dor é de responsabilidade dos profissionais de saúde envolvidos no cuidado de pacientes no perioperatório. Nesse sentido, considera-se importante que os hospitais disponibilizem equipes e procedimentos específicos para alívio da dor no POI, uma vez que os 38 estudos que integraram esta revi- são demonstraram que um manejo adequado pode diminuir doses de fármacos, efeitos colaterais, tempo de hospitalização, otimização da recuperação do paciente e redução dos custos hospitalares.

\section{Categoria 2 - Modalidades não farmacológicas para manejo da dor em pacientes no POI}

Os medicamentos utilizados para tratamento da DPO podem causar inúmeros efeitos adversos com repercussões na qualidade de vida do paciente (HARIRFOROOSH; ASGHAR; JAMALI, 2013; LEPPERT, 2019; TICK et al., 2018). Para alívio da DPO e diminuição dos efeitos adversos, vários métodos não farmacológicos complementares podem ser adotados (KHORSAND et al., 2015; WU et al., 2016). Uma revisão sistemática e meta-análise avaliou acupuntura versus terapia convencional com uso de tratamento farmacológico no tratamento da DPO aguda de cirurgia de costas. O estudo mostrou que a técnica de acupuntura pode ser uma maneira acessível, conveniente, econômica, relativamente segura, eficaz e com raros eventos adversos (CHO et al., 2015).

Artigo de revisão sistemática sobre a eficácia da acupuntura auditiva (EA) para alívio imediato da dor identificou entre os 10 estudos incluídos a presença de eventos adversos em 6 deles, porém eles foram mínimos e transitórios, como dor no local da agulha de acupuntura, tontura e náusea (MURAKAMI; FOX; DIJKERS, 2017). Os autores pontuam que os efeitos da acupuntura mostram-se tão seguros quanto os dos medicamentos alopáticos utilizados para alívio da dor. Desse modo, eles evidenciaram que a EA pode ser uma modalidade promissora para reduzir a dor dentro de 48 horas após a operação, com menos efeitos colaterais que outros medicamentos utilizados atualmente, além de ser acessível e fácil de executar. Em outro estudo com 46 pacientes na Alemanha, verificou-se efeito analgésico significativo da acupuntura no tratamento da dor pós-tonsectomia, e mostrou eficácia como complemento para os Aines (DINGEMANN et al., 2017).

Ainda sobre acupuntura, pesquisa avaliou sua efetividade e técnicas relacionadas no tratamento da DPO (WU et al., 2016). No primeiro dia após a cirurgia, foram realizadas 3 análises: Acupuntura versus controle (diclofenaco e metamizol (dipirona) orais e lidocaína local ou placebo), na qual o grupo experimental apresentou menos dor que o grupo controle, mas ambos usaram doses semelhantes de analgésicos opioides; Eletroacupuntura versus controle (estimulação elétrica nervosa transcutânea simulada (sham) ou nenhuma estimulação elétrica), entre os quais 
não houve diferenças significativa no escore de dor e quantidade cumulativa de analgésicos opioides; e Estimulação de acupuntura elétrica transcutânea (Teas) versus controle (tratamento simulado - sham), que indicou que os pacientes tratados com Teas apresentaram menor dor e menos uso de analgésicos opioides do que o grupo controle. Os autores afirmam que há evidências de que a acupuntura convencional e Teas podem ser mais eficazes do que a eletroacupuntura para manejo da DPO, pois melhoram a DPO 24 horas após a cirurgia.

A dor pós-cesariana é um dos problemas que abala a relação precoce entre mães e recém-nascidos. Um estudo clínico com 80 mulheres em uma enfermaria de obstetrícia, no Irã, avaliou o efeito da massagem de mãos e pés na dor pós-cesariana (ABBASPOOR; AKBARI; NAJAR, 2014). A massagem foi iniciada 1,5-2 horas após o uso de medicamento anestésico espinhal e uma escala analógica visual foi utilizada para determinar a intensidade da dor antes, imediatamente e 90 minutos após 5 minutos de massagem. Os resultados do estudo mostraram que 20 minutos de massagem manual nos pés e mãos reduziu o uso de analgésicos e a intensidade da dor pós-cesariana nos primeiros 90 minutos. Esta técnica, portanto, pode ser considerada um método complementar para reduzir eficazmente a dor, facilitando a recuperação da paciente, reduzindo custos do tratamento e a ansiedade e aumentando a interação entre mãe e filho.

Ensaio clínico com 105 pacientes na unidade de emergência cirúrgica do Hospital Imam Reza de Mashhad, no Irã, avaliou o efeito da massagem reflexológica sobre o alívio da dor após apendicectomia (KHORSAND et al., 2015). Foram estimuladas duas regiões: 1) o ponto Shen Men da orelha, o qual diminui estresse, dor, tensão, ansiedade, depressão e insônia; 2) e o ponto relacionado ao apêndice e à área intestinal localizado no pé direito. Os achados deste estudo sugerem efeito positivo da reflexologia do pé e da oreIha para redução da dor e uso de analgésicos, principalmente quando aplicada simultaneamente nas duas regiões.

Roykulcharoen e Good (2004) testaram uma técnica de relaxamento sistemático do corpo para avaliar a DPO. Os pacientes foram orientados a sentar em posição confortável na cama, olhos fechados, relaxar sucessivamente cada parte principal do corpo, gradativamente, pelos pés, pernas, quadris e cabeça, durante 5 minutos e repetido 3 vezes com respiração suave e relaxada. A dor foi medida antes e 15 minutos durante a recuperação da primeira deambulação após a cirurgia e os pacientes que utilizaram o relaxamento sistemático tiveram menos sofrimento e sensação de dor.

A musicoterapia, também conhecida como atividade que proporciona relaxamento, foi estudada (LIN et al., 2011). Os autores utilizaram músicas suaves, pop, clássicas, sagradas e de sons da natureza, durante três dias antes e dois dias depois da cirurgia, e ocorreu estabilização dos batimentos cardíacos de 60-72 bpm/min. Eles afirmam que a técnica diminuiu a ansiedade, pressão arterial e dor dos participantes e sugerem que a oferta de música pode melhorar o bem-estar do paciente no perioperatório.

Outro estudo investigou efeito da estimulação nervosa elétrica transcutânea de alta frequência (Tens) como terapia complementar para alívio da dor na transição da analgesia epidural (EDA) para geral após cirurgia pancreática (BJERSÅ; ANDERSSON, 2014) e constatou que não houve diferenças na classificação de dor em relação ao grupo que recebeu tratamento simulado (sham - grupo controle). O grupo Tens, entretanto, consumiu uma média de $22,1 \mathrm{mg} /$ paciente de morfina e o grupo controle $37,3 \mathrm{mg} /$ paciente. Os autores concluíram que o referido estudo não encontrou suporte para rejeitar o uso de Tens como complemento durante a transição da anestesia epidural para geral.

Dimitriou et al. (2017) em revisão sistemática analisaram os resultados de todos os ensaios clínicos randomizados que estudaram a eficácia da aromaterapia (AT) como um tratamento complementar da DPO, com as seguintes essências de óleos de lavanda: rosa damascena, eucalipto citriodora e mandarim, e afirmam que, embora a AT seja apresentada como tratamento complementar barato, com menos efeitos adversos, não se pode afirmar que as evidências sejam suficientes para determinar sua eficácia como abordagem não farmacológica.

A análise dos estudos desenvolvidos em diferentes países mostra que os métodos não farmacológicos podem sim se constituírem em uma modalidade complementar promissora para melhorar a DPO, porém requerem mais investigações. A maioria compreende técnicas acessíveis e fáceis de serem executadas, de baixo custo e com menores eventos adversos, porém devem ser praticadas por profissionais habilitados e devidamente capacitados para tal. Evidencia-se lacuna importante de conhecimento de tal assunto em âmbito de Brasil, referentes a estudos que utilizam esta modalidade de tratamento da dor, devendo a pesquisa sobre este assunto ser estimulada. 
Entre as limitações deste estudo destaca-se principalmente o fato de a amostra incluir apenas os artigos disponíveis na íntegra on-line, observando-se que estudos que não seguiram este critério de inclusão e que poderiam trazer contribuição para os resultados encontrados podem ter sido excluídos na etapa de seleção. Além disso, ao usar como critério de seleção preliminar somente a leitura dos títulos e dos resumos dos estudos, algum resultado importante também pode ter sido desconsiderado. Por fim, outra limitação é que somente foram analisados artigos escritos em Português, Espanhol e Inglês.

\section{CONCLUSÃO}

A dor é um sinal vital, portanto requer monitoramento e avaliação, principalmente nos pacientes em POI. Assim, o seu manejo adequado se constitui em um desafio para os profissionais da saúde devido a sua subjetividade, complexidade e multidimensionalidade.

O conhecimento das evidências científicas sobre as modalidades farmacológicas e não farmacológicas, além de propiciar diminuição efetiva da dor, permite a redução das doses de fármacos usadas, os efeitos colaterais, o tempo de hospitalização, além de otimizar a recuperação do paciente e de reduzir custos hospitalares. Assim, este estudo foi importante, pois permitiu a visualização de métodos e conhecimento de evidências tanto referentes ao tratamento farmacológico como ao não farmacológico.

Analisando-se os dados aqui apresentados, concluiu-se que, embora vários medicamentos tenham se mostrado eficazes para diminuir a dor no $\mathrm{POI}$, a terapia multimodal mostrou-se mais eficaz do que quando não utilizados em associação. Em relação à terapia não farmacológica, embora mais estudos ainda sejam necessários para comprovar sua eficácia, eles podem se constituir em uma modalidade complementar promissora para melhorar a DPO.

Por fim, a lacuna existente referente a estudos sobre o uso de medicamentos adequados às condições do paciente e tipo de cirurgia são fundamentais e podem ser aliados ao uso de terapia não farmacológica, como complementar no tratamento da dor.

\section{AGRADECIMENTO}

Este trabalho teve apoio financeiro da Coordenação de Aperfeiçoamento de Pessoal de Nível Superior (Capes).

\section{CONFLITO DE INTERESSES}

Os autores declaram que não existem conflitos de interesses.

\section{REFERÊNCIAS}

ABBASPOOR, Z.; AKBARI, M.; NAJAR, S. Effect of foot and hand massage in post-cesarean section pain control: a randomized control trial. Pain Management Nursing, v. 15, n. 1, p. 132-136, 2014.

ARAIN, S. R. et al. The efficacy of dexmedetomidine versus morphine for postoperative analgesia after major inpatient surgery. Anesthesia and Analgesia, v. 98, p. 153-158, 2004.

ARSLAN, M. et al. Comparison of the analgesic effects of intravenous paracetamol and lornoxicam in postoperative pain following thyroidectomies. Agri, v. 23, n. 4, p. 160-166, 2011.

BARDIN, L. Análise de conteúdo. São Paulo: Edições 70, 2011.

BELL, R. F. et al. Perioperative ketamine for acute postoperative pain. The Cochrane Database of Systematic Review, $\mathrm{v}$. 25, n. 1, 2006.

BJERSÅ, K.; ANDERSSON, T. High frequency TENS as a complement for pain relief in postoperative transition from epidural to general analgesia after pancreatic resection. Complementary Therapies in Clinical Practice, v. 20, n. 1, p. 5-10, 2014.

CHEN, J. Q. et al. Preoperative and postoperative analgesic techniques in the treatment of patients undergoing transabdominal hysterectomy: a preliminary randomized trial. BMC Anesthesioly, v. 15, p. 70. 2015.

$\mathrm{CHO}, \mathrm{Y}$. H. et al. Acupuncture for acute postoperative pain after back surgery: a systematic review and meta-analysis of randomized controlled trials. Pain Practice, v. 15, n. 3, p. 279-291, 2015.

DIMITRIOU, V. et al. The use of aromatherapy for postoperative pain Management: a systematic review of randomized controlled trials. Journal of Peri Anesthesia Nursing, v. 32, n. 6, p. 530-541, 2017.

DINGEMANN, J. et al. Acupuncture in posttonsillectomy pain: A prospective, double-blinded, randomized, controlled trial. HNO, v. 65, n. 1, p. 73-79, 2017.

DIRKMANN, D. et al. Effects of parecoxib on analgesia benefit and blood loss following open prostatectomy: a multicentre randomized trial. BMC Anesthesiology, v. 15, p. 31, 2015.

FARES, K. M. et al. Efficacy and safety of intraperitoneal dexmedetomidine with bupivacaine in laparoscopic colorectal cancer surgery, a randomized trial. Pain Medicine, v. 16, n. 6, p. 1.186-1.194, 2015.

FARSHCHI, A.; GHIASI, G. Comparison the analgesic effects of single dose administration of tramadol or piroxicam on postoperative pain after cesarean delivery. Acta Medica Iran, v. 48, n. 3, p. 148-153, 2010. 
FLETCHER, M. C.; SPERA, J. F. Manejo da dor pós-operatória aguda após a cirurgia bucal. Dental of Clinics North America, 2012.

FREDHEIM, O. M.; BORCHGREVINK, P. C.; KVARSTEIN, G. Post-operative pain management in hospitals. Tidsskrift for den Norske Laegeforening, v. 131, n. 18, p. 1.772-1.776, 2011.

GOKTEN, O. E. et al. Efficacy of levobupivacaine infiltration to nephrosthomy tract in combination with intravenous paracetamol on postoperative analgesia in percutaneous nephrolithotomy patients. Journal of Endourology, v. 25, n. 1, p. 35-39, 2011.

HARIRFOROOSH, S; ASGHAR, W.; JAMALI, F. Advrese effects of nonsteroidal anti-inflammatory drugs: an update of gastrointestinal, cardiovascular and renal complications. Journal of Pharmacy and Pharmaceutical Sciences, v. 16, n. 5, p. 821-847, 2013.

HARTRICK, C. T. et al. Fentanyl iontophoretic transdermal system for acute-pain management after orthopedic surgery: a comparative study with morphine intravenous patient-controlled analgesia. Regional Anesthesia an Pain Medicine, v. 31, n. 6, p. 546-554, 2006.

IUPPEN, L. S.; SAMPAIO, F. H.; STADÑIK, C. M. B. Satisfação dos pacientes com a implantação do conceito dor o quinto sinal vital, no controle da dor pós-operatória. Revista Dor, v. 12, n. 1, p. 29-34, 2011.

KHALILI, G.; JANGHORBANI, M.; SARYAZDI, H. Effect of preemptive and preventive acetaminophen on postoperative pain score: a randomized, double-blind trial of patients undergoing lower extremity surgery. Journal of Clinical Anesthesia, v. 25, n. 3, p. 188-192, 2013.

KHORSAND, A. et al. Evaluation of the Effect of Reflexology on Pain Control and Analgesic Consumption After Appendectomy. Journal of Alternative Complementary Medicine, v. 21, n. 12, p. 774-780, 2015.

KIM, H. C. et al. Efficacy of intrathecal morphine for postoperative pain management following open nephrectomy. Journal of International Medical Research, 2016.

KOMANN, M. et al. Non-pharmacological methods and post-operative pain relief: an observational study. Anesthesiology and Pain Medicine, v. 9, n. 2, p. e84674, 2019.

KOTSOVOLIS, G. et al. Comparison between the combination of gabapentin, ketamine, lornoxicam, and local ropivacaine and each of these drugs alone for pain after laparoscopic cholecystectomy: a randomized trial. Pain Practice, v. 15, n. 4, p. 355-363, 2015.

LEPPERT, W. Pain management with opioid analgesics - How to improve efficacy, while reducing adverse effects and improve patients' quality of life. Current Pharmaceutical Design, v. 25, n. 30, p. 3.195-3.196, 2019.

LIANG, L. et al. The efficiency of intravenous acetaminophen for pain control following total knee and hip arthroplasty: A systematic review and meta-analysis. Medicine. Baltimore v. 96, n. 46, p. 85-86, 2017.
LIN, P. C. et al. Music therapy for patients receiving spine surgery. Journal of Clinical Nursing, v. 20, n. 7-8, p. 960-968, 2011.

MAHDAVI, A. et al. Comparison of morphine suppository and diclofenac suppository for pain management after elective caesarean section. Acta Medicine Iran, v. 54, n. 11, p. 709-712, 2016.

MARTÍNEZ, A. C. et al. Intravenous ibuprofen for treatment of post-operative pain: a multicenter, double blind, placebo-controlled, randomized clinical trial. Plos One, v. 11, n. 5, 2016.

MEISSNER, W. et al. Improving the management of post-operative acute pain: priorities for change. Current Medical Research and Opinion, v. 31, n. 11, p. 2.131-2.143, 2015.

MELNYK, B. M.; FINEOUT-OVERHOLT, E. Evidence based practice in nursing \& healthcare: a guide to best practice. 2 . ed. Philadelphia: Wolters Kluwer Healths, 2011.

MERCANOĞLU, E. et al. Comparison of intravenous morphine, epidural morphine with/ without bupivacaine or ropivacaine in post-thoracotomy pain management with patient controlled analgesia technique. Brazilian Journal of Anesthesiology, v. 63, n. 2, p. 213-219, 2013.

MOREIRA, L. et al. Analgesia no pós-cirúrgico: panorama do controle da dor. Revista Dor, v. 14, n. 2, p. 106-110, 2013.

MORO, T. E.; BRILHA, T. G.; MARIANO, R. K. Implantação de protocolo para avaliação e manuseio da dor aguda na sala de recuperação pós-anestésica de hospital terciário de Sorocaba. Revista da Faculdade de Ciências Médicas de Sorocaba, v. 14, n. 3, p. 95-99, 2012.

MURAKAMI, M.; FOX, L.; DIJKERS, M. P. Ear acupuncture for immediate pain relief-a systematic review and meta-analysis of randomized controlled trials. Pain Medicine, v. 18, n. 3, p. 551-564, 2017.

OHTANI, N. et al. Perioperative infusion of dexmedetomidine at a high dose reduces postoperative analgesic requirements: a randomized control trial. Journal of Anesthesia, v. 25, p. 872-878, 2011.

PARK, M.; JEON, Y. Preoperative pregabalin prolongs duration of spinal anesthesia and reduces early postoperative pain: A double-blind, randomized clinical consorte study. Medicine, Baltimore, v. 95, n. 36, 2016.

PENG, K. et al. Effect of intraoperative dexmedetomidine on post-cranioctomy pain. Clinical Therapeutics, v. 37, n. 5, p. 1.114-1.121, 2015.

PERDREAU, A.; JOUDET, T. Efficacy of multimodal analgesia injection combined with corticosteroids after arthroscopic rotator cuff repair. Orthopaedics \& Traumatology: Surgery \& Research, v. 101, n. 8, p. 337-345, 2015.

PIMENTA, C. A. de M. Alívio da dor: experiências de enfermagem na utilização de técnicas não farmacológicas. Revista Paulista de Enfermagem, v. 9, n. 2, p. 73-77, 1990.

PYO, J. H. et al. A comparative randomized trial on the optimal timing of dexamethasone of for pain relief after endoscopic submucosal dissection for early gastric neoplasm. Gut and Liver, v. 10, n. 4, p. 549-555, 2016. 
ROCHA, S. L.; MORAES, W. M. Assistência de enfermagem no controle da dor na sala de recuperação pós-anestésica. Revista Dor, v. 11, n. 3, p. 254-258, 2010.

ROUTMAN, H. D. et al. Local injection of liposomal bupivacaine combined with intravenous dexamethasone reduces postoperative pain and hospital stay after shoulder arthroplasty. Journal of Shoulder Elbow Surgery, v. 26, n. 4, p. 641647, 2017.

ROYKULCHAROEN, V.; GOOD, M. Systematic relaxation to relieve postoperative pain. Journal of Advanced Nursing, v. 48, n. 2, p. 140-148, 2004.

RUSSO, A. et al. Efficacy of scheduled time cetorolac administration compared to continuous infusion for post-operative pain after abdominal surgery. European Review for Medical Pharmacological Sciences, v. 16, n. 12, p. 1.6751.679, 2012.

SANTOSO, J. T. et al. Multimodal pain control is associated with reduced hospital stay following open abdominal hysterectomy. European Journal of Obstetrics Gynecology and Reproductive Biology, v. 183, p. 48-51, 2014.

SARRIDOU, D. G. et al. Intravenous parecoxib and continuous femoral block for postoperative analgesia after total knee arthroplasty. A randomized, double-blind, prospective trial. Journal, v. 18, n. 3, p. 267-276, 2015.

SMELTZER, S. C. et al. Tratado de enfermagem médico-cirúrgica: conceitos perioperatórios e cuidado de enfermagem. 9. ed. Rio de Janeiro: Guanabara Koogan, 2009.

TAN, M.; LAW, L. S.; GAN, T. J. Optimizing pain management to facilitate enhanced recovery after surgery pathways. $\mathrm{Ca}$ nadian Journal of Anesthesia, v. 62, p. 203-218, 2015.

TICK, H. et al. Evidence-based nonpharmacologic strategies for comprehensive pain care: the consortium pain task force white paper. Explore (NY), v. 14, n. 3, p. 177-211, 2018.

TUNCEL, A. et al. Comparison of different postoperative paın managements in patients submitted to transperitoneal laparoscopic renal and adrenal surgery. International Brazilian Journal of Urology, v. 41, n. 4, p. 669-675, 2015.

VAIMAN, M.; KRAKOVSKI, D.; HAITOV Z. Oxycodone and dexamethasone for pain management after tonsillectomy: a placebo-controlled EMG assessed clinical trial. Medical Sscience Monitor, v. 17, n. 10, p. 25-31, 2011.

WHITE, S. et al. Impact of liposomal bupivacaine administration on postoperative pain in patients undergoing total knee replacement. Pharmacotherapy, v. 35, n. 5, p. 477481, 2015.

WU, M. S. et al. The efficacy of acupuncture in post-operative pain management: a systematic review and meta-analysis. Plos One, v. 11, n. 3, 2016.

YAO, Z.; SHEN, C.; ZHONG, Y. perioperative pregabalin for acute pain after gynecological surgery: a meta-analysis. Clinical Therapeutics, v. 37, n. 5, p. 1.128-1.135, 2015.

ZHANG, Y. L. et al. Effect of intraoperative high-dose remifentanil on postoperative pain: a prospective, double blind, randomized clinical trial. Plos One, v. 9, n. 3, 2014. 
Material Suplementar: Quadro comparativo dos estudos avaliados ( $\mathrm{n}=38$ )

\section{TRATAMENTO FARMACOLÓGICO PARA MANEJO DA DOR EM PÓS-OPERATÓRIO IMEDIATO}

\begin{tabular}{|c|c|c|c|}
\hline \begin{tabular}{|l|} 
Autores/ periódico/ \\
ano de publicação
\end{tabular} & \begin{tabular}{|c|}
$\begin{array}{c}\text { Tipo de estudo/ } \\
\text { amostra }\end{array}$ \\
\end{tabular} & Objetivos & Principais resultados/Conclusão \\
\hline \multicolumn{4}{|l|}{ Nível de evidência: 1} \\
\hline \begin{tabular}{|l|} 
Yao, Z., Shen, C., \\
Zhong, Y. \\
Clinical Therapeutics \\
(2015)
\end{tabular} & \begin{tabular}{|l|} 
Meta-análise de \\
ensaios clínicos \\
$(\mathrm{n}=452)$
\end{tabular} & $\begin{array}{l}\text { Avaliar a capacidade da pregablina no } \\
\text { controle de dor pós-operatória aguda } \\
\text { após a cirurgia ginecológica. }\end{array}$ & $\begin{array}{l}\text { A pregabalina teve efeito analgésico e } \\
\text { poupador de opioides e não aumenta a } \\
\text { frequência de efeitos adversos. }\end{array}$ \\
\hline \begin{tabular}{|l|} 
Bell, R. F. et al. \\
Acta \\
Anaesthesiologica \\
Scandinavica (2005) \\
\end{tabular} & $\begin{array}{l}\text { Revisão } \\
\text { sistemática ( } n= \\
37 \text { ensaios) }\end{array}$ & $\begin{array}{l}\text { Avaliar a eficácia e tolerabilidade } \\
\text { da cetamina administrada } \\
\text { perioperatoriamente no tratamento } \\
\text { de dor pós-operatória aguda em } \\
\text { adultos. }\end{array}$ & $\begin{array}{l}\text { Nas primeiras } 24 \text { horas após a cirurgia, } \\
\text { a cetamina reduziu o uso de morfina, } \\
\text { náusea e vômito pós-operatório. Os efeitos } \\
\text { adversos foram leves ou ausentes. }\end{array}$ \\
\hline $\begin{array}{l}\text { Liang, L. et al. } \\
\text { Medicine (2017) }\end{array}$ & $\begin{array}{l}\text { Meta-análise } \\
(n=865)\end{array}$ & \begin{tabular}{|l|} 
Avaliar a eficácia e segurança \\
do paracetamol intravenoso \\
como complemento da analgesia \\
multimodal para controle da dor após \\
artroplastia total da articulação. \\
\end{tabular} & $\begin{array}{l}\text { O paracetamol intravenoso foi eficaz para } \\
\text { reduzir a dor pós-operatória e o consumo } \\
\text { de opioide ainda comparado ao placebo. }\end{array}$ \\
\hline $\begin{array}{l}\text { Fredheim, O.M., } \\
\text { Borchgrevink, P.C., } \\
\text { Kvarstein, G. } \\
\text { Tidsskrift for } \\
\text { Den Norske } \\
\text { Laegeforening } \\
\text { (2011) } \\
\end{array}$ & Revisão & $\begin{array}{l}\text { Discutir indicações, contraindicações } \\
\text { e a eficácia das várias modalidades } \\
\text { de tratamento farmacológico } \\
\text { multimodal. }\end{array}$ & $\begin{array}{l}\text { Várias modalidades de tratamento são } \\
\text { eficazes para a dor pós-operatória, mas } \\
\text { é importante que o tratamento esteja } \\
\text { bem organizado e que inclua rotinas para } \\
\text { avaliação sistemática da dor. }\end{array}$ \\
\hline \multicolumn{4}{|l|}{ Nível de evidência: 2} \\
\hline $\begin{array}{l}\text { Hartrick, C. T. et al. } \\
\text { Regional Anesthesia } \\
\text { and Pain Medicine } \\
\text { (2006) }\end{array}$ & \begin{tabular}{|l|} 
Estudo clínico \\
randomizado \\
de fase III, \\
multicêntrico, \\
aberto e \\
controlado ativo \\
( $\mathrm{n}=799)$ \\
\end{tabular} & $\begin{array}{l}\text { Comparar a eficácia, segurança } \\
\text { e conveniência do Fentanil } \\
\text { transdérmico (ITS) com morfina } \\
\text { intravenosa analgésica controlada } \\
\text { pelo paciente para o tratamento da } \\
\text { dor aguda após a reposição unilateral } \\
\text { total do quadril. }\end{array}$ & $\begin{array}{l}\text { Ambas as técnicas foram semelhantes } \\
\text { para o controle da dor pós-operatória nas } \\
\text { primeiras } 24 \text { horas de tratamento. }\end{array}$ \\
\hline $\begin{array}{l}\text { Farshchi, A., Ghiasi, } \\
\text { L. } \\
\text { Acta Medica Iranica } \\
\text { (2010) }\end{array}$ & $\begin{array}{l}\text { Estudo clínico } \\
\text { randomizado, } \\
\text { duplo-cego }(n= \\
150)\end{array}$ & \begin{tabular}{|l|} 
Investigar os efeitos comparativos \\
de doses de Tramadol (100 mg) e \\
Piroxicam ( $20 \mathrm{mg}$ ) no manejo da dor \\
pós-operatório em mulheres em parto \\
eletivo por cesariana.
\end{tabular} & $\begin{array}{l}\text { As injeções de } 20 \mathrm{mg} \text { de piroxicam em } \\
\text { dose única aliviaram a dor após cesariana, } \\
\text { bem como o tramadol e pode reduzir os } \\
\text { requisitos de analgésicos opioides com } \\
\text { menos efeitos colaterais adversos durante } \\
\text { as primeiras } 24 \text { horas. }\end{array}$ \\
\hline $\begin{array}{l}\text { Gokten, O. E. et al. } \\
\text { Journal of } \\
\text { Endourology (2011) }\end{array}$ & $\begin{array}{l}\text { Estudo clínico } \\
\text { randomizado } \\
(\mathrm{n}=66)\end{array}$ & \begin{tabular}{|l|} 
Avaliar a eficácia da infiltração \\
anestésica intraoperatória de \\
levobupivacaína local em combinação \\
com infusão intravenosa de \\
paracetamol no tratamento da \\
dor pós-operatória em pacientes \\
submetidos à nefrolitotomia \\
percutânea. \\
\end{tabular} & $\begin{array}{l}\text { Esta combinação mostrou-se segura e } \\
\text { eficaz como método analgésico após } \\
\text { nefrolitotomia percutânea. }\end{array}$ \\
\hline $\begin{array}{l}\text { Arslan, M. et al. } \\
\text { Agriculture (2011) }\end{array}$ & $\begin{array}{l}\text { Estudo clínico } \\
\text { randomizado } \\
(\mathrm{n}=60)\end{array}$ & $\begin{array}{l}\text { Determinar a eficácia do paracetamol } \\
\text { e lornoxicam intravenoso na analgesia } \\
\text { pós-operatória e redução do } \\
\text { tramadol. }\end{array}$ & $\begin{array}{l}\text { A administração desses medicamentos } \\
\text { após cirurgia de tireoide diminuiu o escore } \\
\text { de dor pós-operatória e o uso de opioides, } \\
\text { bem como a incidência de náuseas e } \\
\text { vômitos, além de prolongar o tempo para o } \\
\text { primeiro suplemento analgésico. }\end{array}$ \\
\hline
\end{tabular}




\begin{tabular}{|c|c|c|c|}
\hline $\begin{array}{l}\text { Russo, A. et al. } \\
\text { European Review } \\
\text { for Medical and } \\
\text { Pharmacological } \\
\text { Sciences (2012) }\end{array}$ & $\begin{array}{l}\text { Ensaio clínico } \\
\text { randomizado } \\
(\mathrm{n}=80)\end{array}$ & $\begin{array}{l}\text { Avaliar se houve diferença no alívio } \\
\text { da dor pós-operatória quando o } \\
\text { cetorolaco foi administrado com } \\
\text { infusão contínua ou em horários pré- } \\
\text { estabelecidos. }\end{array}$ & $\begin{array}{l}\text { A administração de cetorolaco em horários } \\
\text { preestabelecidos, a cada } 8 \text { horas, oferece } \\
\text { maiores benefícios em relação à sua infusão } \\
\text { contínua. }\end{array}$ \\
\hline $\begin{array}{l}\text { Mercanô̂lu, E. et al. } \\
\text { Revista Brasileira } \\
\text { de Anestesiologia } \\
\text { (2013) }\end{array}$ & $\begin{array}{l}\text { Estudo clínico } \\
\text { randomizado, } \\
\text { duplo-cego e } \\
\text { prospectivo ( } \mathrm{n}= \\
60 \text { ) }\end{array}$ & $\begin{array}{l}\text { Determinar a eficácia e os efeitos } \\
\text { colaterais da administração } \\
\text { intravenosa e epidural de morfina, } \\
\text { bupivacaína e ropivacaína no } \\
\text { tratamento da dor pós-toracotomia. }\end{array}$ & $\begin{array}{l}\text { Morfina administrada por via epidural foi } \\
\text { mais eficaz do que por via intravenosa. } \\
\text { A eficácia foi maior no grupo morfina } \\
\text { epidural no pós-operatório tardio e no } \\
\text { grupo morfina epidural + bupivacaína no } \\
\text { período pós-operatório inicial. A morfina } \\
\text { administrada por via epidural foi mais eficaz } \\
\text { e preferida. }\end{array}$ \\
\hline $\begin{array}{l}\text { Zhang, Y. L. et al. } \\
\text { PLoS One (2014) }\end{array}$ & $\begin{array}{l}\text { Ensaio clínico } \\
\text { randomizado, } \\
\text { prospectivo, } \\
\text { duplo cego ( } n= \\
60)\end{array}$ & $\begin{array}{l}\text { Verificar se a administração } \\
\text { intraoperatória de altas doses de } \\
\text { remifentanil pode atenuar a dor pós- } \\
\text { operatória. }\end{array}$ & $\begin{array}{l}\text { A administração intraoperatória de } \\
\text { remifentanil com altas doses diminuiu os } \\
\text { escores da escala visual da dor e morfina } \\
\text { no pós-operatório. Assim, a modulação } \\
\text { de opiáceos intraoperatórios pode ser um } \\
\text { método simples e eficaz de tratamento da } \\
\text { dor no pós-operatório. }\end{array}$ \\
\hline $\begin{array}{l}\text { Tuncel, A. et al. } \\
\text { International } \\
\text { Brazilian Journal Of } \\
\text { Urology (2015) }\end{array}$ & $\begin{array}{l}\text { Estudo clínico } \\
\text { randomizado } \\
(\mathrm{n}=66)\end{array}$ & $\begin{array}{l}\text { Comparar os efeitos da infiltração } \\
\text { local de levobupivacaína, paracetamol } \\
\text { intravenoso, lornoxicam intravenoso } \\
\text { em analgesia pós-operatória em } \\
\text { pacientes submetidos à cirurgia } \\
\text { laparoscópica renal e adrenal } \\
\text { transperitoneal. }\end{array}$ & $\begin{array}{l}\text { O grupo tratado com levobupivacaína } \\
\text { necessitou significativamente mais } \\
\text { tramadol intravenoso quando comparados } \\
\text { com grupos de paracetamol e lornoxicam. }\end{array}$ \\
\hline $\begin{array}{l}\text { Perdreau, A., Joudet, } \\
\text { T. } \\
\text { Orthopaedics \& } \\
\text { Traumatology: } \\
\text { Surgery \& Research } \\
\text { (2015) }\end{array}$ & $\begin{array}{l}\text { Estudo } \\
\text { prospectivo } \\
\text { randomizado } \\
(n=50)\end{array}$ & $\begin{array}{l}\text { Avaliar a eficácia e a segurança } \\
\text { a curto prazo da injeção de } \\
\text { analgésicos multimodais associada } \\
\text { a corticosteroides na cirurgia } \\
\text { artroscópica do manguito rotador. }\end{array}$ & $\begin{array}{l}\text { Esta técnica proporcionou benefício } \\
\text { imediato em termos de analgesia } \\
\text { minimizando o uso de morfina, sem risco } \\
\text { aparente de infecção. }\end{array}$ \\
\hline $\begin{array}{l}\text { Chen, J. Q. et al. } \\
\text { BMC Anesthesiology } \\
\text { (2015) }\end{array}$ & $\begin{array}{l}\text { Estudo } \\
\text { randomizado } \\
(n=100)\end{array}$ & $\begin{array}{l}\text { Investigar os efeitos da analgesia } \\
\text { preventiva multimodal no manejo } \\
\text { da dor, resposta ao estresse e } \\
\text { fatores inflamatórios de pacientes } \\
\text { submetidos à histerectomia } \\
\text { transabdominal. }\end{array}$ & $\begin{array}{l}\text { A analgesia pré-operatória multimodal } \\
\text { reduziu significativamente o escore da } \\
\text { escala visual analógica, inibiu a resposta ao } \\
\text { estresse e reduziu a resposta inflamatória } \\
\text { nestas pacientes, o que pode ser uma } \\
\text { estratégia racional para o controle da dor. }\end{array}$ \\
\hline $\begin{array}{l}\text { Kotsovolis, G. et al. } \\
\text { Pain Practice (2015) }\end{array}$ & $\begin{array}{l}\text { Estudo } \\
\text { randomizado } \\
\text { controlado }(\mathrm{n}= \\
148)\end{array}$ & $\begin{array}{l}\text { Testar se a combinação de } \\
\text { gabapentina ( } 600 \text { mg } 4 \mathrm{~h} \text { antes da } \\
\text { cirurgia, } 600 \mathrm{mg} \text { após } 24 \text { horas), } \\
\text { cetamina (0,3 mg/kg antes da } \\
\text { anestesia), lornoxicam ( } 8 \mathrm{mg} \\
\text { antes da anestesia e } 8 \mathrm{mg} / 12 \mathrm{~h} \text { ) e } \\
\text { ropivacaína local ( } 5 \mathrm{~mL} 7,5 \% \text { nos } \\
\text { locais de inserção) fornece analgesia } \\
\text { superior a cada uma dessas drogas } \\
\text { isoladas nas primeiras } 24 \text { horas após a } \\
\text { colecistectomia laparoscópica. }\end{array}$ & $\begin{array}{l}\text { Esta combinação não proporcionou } \\
\text { analgesia superior à da gabapentina ou } \\
\text { ao lornoxicam isolados. A combinação } \\
\text { reduziu apenas a frequência de náuseas } \\
\text { pós-operatórias, mas mais estudos são } \\
\text { necessários para resultados mais seguros. }\end{array}$ \\
\hline $\begin{array}{l}\text { Fares, K. M. et al. } \\
\text { Pain Medicine } \\
\text { (2015) }\end{array}$ & $\begin{array}{l}\text { Ensaio clínico } \\
\text { randomizado } \\
(\mathrm{n}=45)\end{array}$ & $\begin{array}{l}\text { Investigar a eficácia e a segurança da } \\
\text { dexmedetomidina intraperitoneal } \\
\text { combinada com bupivacaína em } \\
\text { pacientes submetidos à cirurgia } \\
\text { laparoscópica de câncer colorretal. }\end{array}$ & $\begin{array}{l}\text { A administração intraperitoneal de } \\
\text { dexmedetomidina } 1 \mu \mathrm{g} / \mathrm{kg} \text { combinada } \\
\text { com a bupivacaína melhorou a qualidade } \\
\text { e a duração do analgésico pós-operatório. } \\
\text { Proporcionou efeito estimulante analgésico } \\
\text { em comparação com a bupivacaína isolada, } \\
\text { sem efeitos adversos significativos. }\end{array}$ \\
\hline
\end{tabular}




\begin{tabular}{|c|c|c|c|}
\hline $\begin{array}{l}\text { Sarridou, D. G. et al. } \\
\text { Pain Physician } \\
(2015)\end{array}$ & $\begin{array}{l}\text { Estudo clínico } \\
\text { randomizado, } \\
\text { duplo-cego, } \\
\text { prospectivo ( } \mathrm{n}= \\
\text { 90) }\end{array}$ & $\begin{array}{l}\text { Examinar a eficácia analgésica e } \\
\text { diminuição no uso de opioides } \\
\text { do parecoxibe intravenoso em } \\
\text { combinação com bloqueio femoral } \\
\text { contínuo. }\end{array}$ & $\begin{array}{l}\text { Esta combinação proporcionou eficácia } \\
\text { analgésica superior e diminuiu o uso } \\
\text { de opioides em pacientes submetidos à } \\
\text { artroplastia total de joelho. }\end{array}$ \\
\hline $\begin{array}{l}\text { Mahdavi, A. et al. } \\
\text { Acta Medica Iranica } \\
\text { (2016) }\end{array}$ & $\begin{array}{l}\text { Ensaio clínico } \\
\text { randomizado } \\
(\mathrm{n}=100)\end{array}$ & $\begin{array}{l}\text { Investigar a eficácia e os efeitos } \\
\text { colaterais do supositório de morfina } \\
\text { em comparação com o supositório de } \\
\text { diclofenaco para o tratamento da dor } \\
\text { após cirurgia de cesariana. }\end{array}$ & $\begin{array}{l}\text { O supositório de diclofenaco diminuiu os } \\
\text { escores de dor subjetivos nas primeiras } 12 \\
\text { horas, com estatística significativa. }\end{array}$ \\
\hline $\begin{array}{l}\text { Park, M., Jeon, Y. } \\
\text { Medicine (2016) }\end{array}$ & $\begin{array}{l}\text { Estudo clínico } \\
\text { randomizado, } \\
\text { prospectivo e } \\
\text { duplo-cego ( } \mathrm{n}= \\
44)\end{array}$ & $\begin{array}{l}\text { Avaliar a eficácia de uma única dose } \\
\text { de pregabalina em termos de duração } \\
\text { do bloqueio da coluna vertebral e seu } \\
\text { potencial efeito poupador de opioides } \\
\text { durante as primeiras } 24 \text { horas após a } \\
\text { cirurgia urogenital. }\end{array}$ & $\begin{array}{l}\text { Dose única de } 150 \text { mg de pregabalina } \\
\text { antes da cirurgia promoveu a eficácia } \\
\text { da bupivacaína intratecal e melhorou a } \\
\text { analgesia pós-operatória. }\end{array}$ \\
\hline $\begin{array}{l}\text { Gago, M. A. et al. } \\
\text { PLoS One (2016) }\end{array}$ & $\begin{array}{l}\text { Ensaio clínico } \\
\text { randomizado, } \\
\text { multicêntrico, } \\
\text { duplo cego, } \\
\text { controlado por } \\
\text { placebo }(n=206)\end{array}$ & $\begin{array}{l}\text { Avaliar a eficácia e segurança de } \\
\text { uma nova formulação de ibuprofeno } \\
\text { intravenoso para o tratamento da dor } \\
\text { pós-operatória em uma população } \\
\text { europeia. }\end{array}$ & $\begin{array}{l}\text { A administração perioperatória de } \\
\text { ibuprofeno intravenso } 800 \text { mg a cada } 6 \\
\text { horas em pacientes com cirurgia abdominal } \\
\text { diminui o uso de morfina, a dor e o risco } \\
\text { de tratamento de resgate com outros } \\
\text { analgésicos. O ibuprofeno foi seguro e bem } \\
\text { tolerado e pode ser um adjuvante útil para } \\
\text { uso em curto prazo. }\end{array}$ \\
\hline $\begin{array}{l}\text { Pyo, J. H. et al. } \\
\text { Gut and Liver (2016) }\end{array}$ & $\begin{array}{l}\text { Estudo clínico } \\
\text { randomizado } \\
(n=40)\end{array}$ & $\begin{array}{l}\text { Comparar os efeitos clínicos da } \\
\text { dexametasona pré-operatória e pós- } \\
\text { operatória em dor após a dissecção } \\
\text { submucosal endoscópica para } \\
\text { neoplasia gástrica precoce. }\end{array}$ & $\begin{array}{l}\text { A dexametasona pré-operatória produziu } \\
\text { efeito analgésico superior em comparação } \\
\text { com a administração pós-operatória de } \\
\text { dexametasona. }\end{array}$ \\
\hline $\begin{array}{l}\text { Kim, H. C. et al. } \\
\text { Journal of } \\
\text { International } \\
\text { Medical Research } \\
\text { (2016) }\end{array}$ & $\begin{array}{l}\text { Estudo clinico } \\
\text { randomizado } \\
(\mathrm{n}=45)\end{array}$ & $\begin{array}{l}\text { Avaliar a eficácia da morfina intratecal } \\
\text { para controle de dor pós-operatória } \\
\text { em pacientes com carcinoma } \\
\text { de células renais submetidos à } \\
\text { nefrectomia aberta. }\end{array}$ & $\begin{array}{l}\text { A morfina intratecal foi associada a uma } \\
\text { maior analgesia sem complicações graves } \\
\text { quando comparada com a analgesia } \\
\text { endovenosa. }\end{array}$ \\
\hline \multicolumn{4}{|l|}{ Nível de evidência: 3} \\
\hline $\begin{array}{l}\text { Vaiman, M., } \\
\text { Krakovski, D., Haitov, } \\
\text { Z. } \\
\text { Medical Science } \\
\text { Monitor (2011) }\end{array}$ & $\begin{array}{l}\text { Estudo clínico } \\
(n=90)\end{array}$ & $\begin{array}{l}\text { Avaliar a eficácia e segurança } \\
\text { de oxicodona e dexametasona } \\
\text { no tratamento da dor após } \\
\text { amigdalectomia. }\end{array}$ & $\begin{array}{l}\text { A aplicação de oxicodona reduziu } \\
\text { significativamente a dor pós-operatória. A } \\
\text { aplicação de dexametasona é aconselhável } \\
\text { devido à redução da morbidade pós- } \\
\text { operatória, enquanto a redução da dor } \\
\text { pós-operatória é secundária à redução do } \\
\text { edema. }\end{array}$ \\
\hline \multicolumn{4}{|l|}{ Nível de evidência: 4} \\
\hline \begin{tabular}{|l|} 
Santoso, J. T. et al. \\
European Journal \\
of Obstetrics \\
Gynecology and \\
Reproductive (2014) \\
\end{tabular} & $\begin{array}{l}\text { Estudo de } \\
\text { coorte }(n=218)\end{array}$ & \begin{tabular}{|l|} 
Estudar a associação de um protocolo \\
de dor multimodal e redução \\
da internação hospitalar após a \\
histerectomia abdominal aberta.
\end{tabular} & $\begin{array}{l}\text { O controle da dor multimodal reduziu } \\
\text { significativamente o tempo de } \\
\text { hospitalização após o procedimento. }\end{array}$ \\
\hline $\begin{array}{l}\text { White, S. et al. } \\
\text { Pharmacotherapy } \\
(2015)\end{array}$ & $\begin{array}{l}\text { Estudo } \\
\text { de coorte } \\
\text { retrospectivo } \\
(n=120)\end{array}$ & $\begin{array}{l}\text { Determinar se a bupivacaína } \\
\text { lipossomal tem impacto na dor pós- } \\
\text { operatória em pacientes submetidos à } \\
\text { artroplastia total de joelho. }\end{array}$ & $\begin{array}{l}\text { A bupivacaína lipossomal não melhorou } \\
\text { o controle da dor quando comparado } \\
\text { com as estratégias históricas de manejo. } \\
\text { No entanto, as diferenças podem ter sido } \\
\text { influenciadas pelo aumento da utilização de } \\
\text { analgésicos associados entre os pacientes } \\
\text { do grupo controle. }\end{array}$ \\
\hline
\end{tabular}




\begin{tabular}{|l|l|l|l|}
\hline $\begin{array}{l}\text { Routman, H. D. et al. } \\
\text { Journal of Shoulder } \\
\text { and Elbow Surgery } \\
(2017)\end{array}$ & $\begin{array}{l}\text { Estudo de } \\
\text { coorte }(n=55)\end{array}$ & $\begin{array}{l}\text { Avaliar o efeito de bupivacaína } \\
\text { lipossomal e dexametasona } \\
\text { intraoperatória intravenosa durante a a } \\
\text { artroplastia do ombro. }\end{array}$ & $\begin{array}{l}\text { Os pacientes que receberam dexametasona } \\
\text { intravenosa intraoperatória e uma injeção } \\
\text { na ferida com bupivacaína lipossomal, } \\
\text { solução salina e marcaína diminuíram o } \\
\text { período da hospitalização, a necessidade } \\
\text { de narcóticos para dor, conforme escala } \\
\text { visual analógica. Esta combinação de } \\
\text { medicamentos deve ser considerada em } \\
\text { pacientes com artroplastia de ombro como } \\
\text { parte de um programa de dor multimodal. }\end{array}$ \\
\hline
\end{tabular}

TRATAMENTO NÃO FARMACOLÓGICO PARA MANEJO DA DOR EM PÓS-OPERATÓRIO IMEDIATO

\begin{tabular}{|c|c|c|c|}
\hline $\begin{array}{l}\text { Autores, periódico e } \\
\text { ano de publicação }\end{array}$ & \begin{tabular}{|c} 
Tipo de estudo/ \\
amostra
\end{tabular} & Objetivos & Principais resultados/Conclusão \\
\hline \multicolumn{4}{|l|}{ Nível de evidência: 1} \\
\hline $\begin{array}{l}\text { Cho, Y. H. et al. } \\
\text { Pain practice (2015) }\end{array}$ & $\begin{array}{l}\text { Revisão } \\
\text { sistemática e } \\
\text { meta-análise } \\
\text { de ensaios } \\
\text { controlados } \\
\text { aleatórios }(n=3) \\
\end{array}$ & $\begin{array}{l}\text { Avaliar a eficácia do tratamento de } \\
\text { acupuntura para dor pós-operatória } \\
\text { aguda ( } \leq 1 \text { semana) após a cirurgia de } \\
\text { costas. }\end{array}$ & $\begin{array}{l}\text { O estudo apresentou evidências } \\
\text { encorajadoras, mas limitadas, da eficácia } \\
\text { do tratamento de acupuntura para dor pós- } \\
\text { operatória aguda após a cirurgia de costas. }\end{array}$ \\
\hline $\begin{array}{l}\text { Wu, M. S. et al. } \\
\text { PLOS ONE (2016) }\end{array}$ & $\begin{array}{l}\text { Revisão } \\
\text { sistemática e } \\
\text { meta-análise } \\
(\mathrm{n}=3)\end{array}$ & $\begin{array}{l}\text { Avaliar a eficácia da acupuntura e } \\
\text { técnicas relacionadas à acupuntura no } \\
\text { tratamento da dor pós-operatória. }\end{array}$ & $\begin{array}{l}\text { Acupuntura melhorou a dor pós-operatória } \\
\text { no primeiro dia após a cirurgia e reduziu o } \\
\text { uso de opioides. }\end{array}$ \\
\hline $\begin{array}{l}\text { Dimitriou, V. et al. } \\
\text { Journal of } \\
\text { Perianesthesia } \\
\text { Nursing (2016) }\end{array}$ & $\begin{array}{l}\text { Revisão } \\
\text { sistemática } \\
\text { dos ensaios } \\
\text { controlados } \\
\text { aleatórios ( } n= \\
644)\end{array}$ & $\begin{array}{l}\text { Analisar os resultados de todos os } \\
\text { ensaios clínicos randomizados que } \\
\text { estudaram a eficácia da aromaterapia } \\
\text { como um tratamento complementar } \\
\text { para a dor pós-operatória. }\end{array}$ & $\begin{array}{l}\text { Embora a aromaterapia seja apresentada } \\
\text { como um tratamento complementar barato } \\
\text { com uma baixa taxa de efeitos adversos } \\
\text { que melhora a satisfação do paciente, não } \\
\text { se pode determinar que existam provas } \\
\text { suficientes para concluir sua eficácia } \\
\text { quanto à abordagem não farmacológica } \\
\text { para a redução da intensidade da dor pós- } \\
\text { operatória. }\end{array}$ \\
\hline $\begin{array}{l}\text { Murakami, M., Fox, } \\
\text { L., Dijkers, M. } \\
\text { Pain Medcine (2016) }\end{array}$ & $\begin{array}{l}\text { Revisão } \\
\text { sistemática }(n= \\
10)\end{array}$ & $\begin{array}{l}\text { Revisar sistematicamente a literatura } \\
\text { sobre a eficácia da acupuntura } \\
\text { auditiva para alívio imediato da dor. }\end{array}$ & $\begin{array}{l}\text { A acupuntura da orelha pode ser uma } \\
\text { modalidade promissora para ser usada para } \\
\text { reduzir a dor dentro de } 48 \text { horas, com um } \\
\text { perfil de efeito secundário baixo. }\end{array}$ \\
\hline \multicolumn{4}{|l|}{ Nível de evidência: 2} \\
\hline $\begin{array}{l}\text { Roykulcharoen, V., } \\
\text { Good, M. } \\
\text { Journal of Advanced } \\
\text { Nursing (2004) }\end{array}$ & $\begin{array}{l}\text { Ensaio clínico } \\
\text { controlado } \\
\text { randomizado } \\
\text { com grupos }(\mathrm{n}= \\
102)\end{array}$ & $\begin{array}{l}\text { Examinar os efeitos de um método } \\
\text { sistemático de relaxar o corpo nos } \\
\text { componentes sensoriais e afetivos } \\
\text { da dor pós-operatória, ansiedade } \\
\text { e ingestão de opioides após a } \\
\text { emboscada inicial. }\end{array}$ & $\begin{array}{l}\text { Reduções na sensação e angústia da dor } \\
\text { foram encontradas quando os pacientes } \\
\text { pós-operatórios usaram relaxamento } \\
\text { sistemático. }\end{array}$ \\
\hline $\begin{array}{l}\text { Lin, P. C. et al. } \\
\text { Journal of Clinical } \\
\text { Nursing (2011) }\end{array}$ & $\begin{array}{l}\text { Estudo clínico } \\
\text { randomizado } \\
(n=60)\end{array}$ & $\begin{array}{l}\text { Avaliar os efeitos da terapia musical } \\
\text { na ansiedade, dor pós-operatória e } \\
\text { reações fisiológicas ao sofrimento } \\
\text { emocional e físico em pacientes } \\
\text { submetidos à cirurgia da coluna } \\
\text { vertebral. }\end{array}$ & $\begin{array}{l}\text { A terapia musical tem efeitos positivos } \\
\text { sobre os níveis de ansiedade e dor em } \\
\text { pacientes submetidos à cirurgia da coluna } \\
\text { vertebral. }\end{array}$ \\
\hline $\begin{array}{l}\text { He, B. J. et al. } \\
\text { Pain Medicine } \\
(2013)\end{array}$ & $\begin{array}{l}\text { Estudo clinico } \\
\text { randomizado } \\
\text { prospectivo }(n= \\
90)\end{array}$ & $\begin{array}{l}\text { Examinar se a acupressão auricular } \\
\text { pode aliviar a dor pós-operatória e } \\
\text { diminuir o consumo de narcóticos } \\
\text { e seus efeitos adversos para os } \\
\text { pacientes após a artroplastia total do } \\
\text { joelho. }\end{array}$ & $\begin{array}{l}\text { A acupressão aliviou a dor pós-operatória, } \\
\text { diminuiu o consumo de opioides e seus } \\
\text { efeitos adversos e promoveu a reabilitação } \\
\text { precoce. Além disso, esta intervenção tem } \\
\text { a vantagem de menores custos, menos } \\
\text { complicações, aplicação simples e alta } \\
\text { segurança. }\end{array}$ \\
\hline
\end{tabular}




\begin{tabular}{|c|c|c|c|}
\hline $\begin{array}{l}\text { Bjerså, K., } \\
\text { Andersson, T. } \\
\text { Complementary } \\
\text { Therapies in Clinical } \\
\text { Practice (2014) }\end{array}$ & $\begin{array}{l}\text { Estudo clínico } \\
\text { randomizado } \\
\text { prospectivo, } \\
\text { único cego ( } \mathrm{n}= \\
55 \text { ) }\end{array}$ & $\begin{array}{l}\text { Investigar o efeito da estimulação } \\
\text { nervosa elétrica transcutânea de } \\
\text { alta frequência (Tens) como um } \\
\text { tratamento para aliviar a dor na } \\
\text { transição da analgesia epidural (EDA) } \\
\text { para a analgesia geral após a cirurgia } \\
\text { pancreática por incisão horizontal e } \\
\text { abdominal. }\end{array}$ & $\begin{array}{l}\text { Não foi encontrado suporte para rejeitar o } \\
\text { uso de Tens como complemento durante } \\
\text { a transição da analgesia epidural para } \\
\text { analgesia geral. }\end{array}$ \\
\hline $\begin{array}{l}\text { Abbaspoor, Z., } \\
\text { Akbari, M., Najar, S. } \\
\text { Pain Management } \\
\text { Nursing (2014) }\end{array}$ & $\begin{array}{l}\text { Estudo clínico } \\
\text { randomizado e } \\
\text { controlado }(n= \\
80)\end{array}$ & $\begin{array}{l}\text { Determinar o efeito da massagem } \\
\text { de mão e pé na dor na seção pós- } \\
\text { cesariana. }\end{array}$ & $\begin{array}{l}\text { A massagem do pé e da mão pode } \\
\text { ser considerada como um método } \\
\text { complementar para reduzir a dor da } \\
\text { cesariana e reduzir a quantidade de } \\
\text { medicamentos e seus efeitos colaterais. }\end{array}$ \\
\hline $\begin{array}{l}\text { Dingemann, J. et al. } \\
\text { HNO (2017) }\end{array}$ & $\begin{array}{l}\text { Estudo } \\
\text { prospectivo, } \\
\text { duplo-cego, } \\
\text { randomizado e } \\
\text { controlado ( } \mathrm{n}= \\
46)\end{array}$ & $\begin{array}{l}\text { Avaliar o efeito da acupuntura como } \\
\text { uma terapia adicional na gestão da } \\
\text { dor pós-tonsilectomia. }\end{array}$ & $\begin{array}{l}\text { A acupuntura é um complemento eficaz } \\
\text { para os Aines no tratamento da dor pós- } \\
\text { tonsectomia e pacientes com alergias, } \\
\text { intolerância a drogas ou resposta } \\
\text { reduzida aos medicamentos comumente } \\
\text { administrados podem se beneficiar da } \\
\text { acupuntura. }\end{array}$ \\
\hline \multicolumn{4}{|l|}{ Nível de evidência: 3} \\
\hline $\begin{array}{l}\text { Khorsand, A et al. } \\
\text { Journal of } \\
\text { Alternative and } \\
\text { Complementary } \\
\text { Medicine (2015) }\end{array}$ & $\begin{array}{l}\text { Ensaio clínico } \\
\text { randomizado } \\
(\mathrm{n}=105)\end{array}$ & $\begin{array}{l}\text { Determinar o efeito da massagem } \\
\text { reflexológica sobre o alívio da dor } \\
\text { após a apendicectomia. }\end{array}$ & $\begin{array}{l}\text { A reflexologia é eficaz para reduzir a dor } \\
\text { após a cirurgia de apendicectomia. }\end{array}$ \\
\hline
\end{tabular}

